\title{
Spamex: A Multi Array Methodology to Detect Computerized Spammers in Twitter Social Media
}

\author{
Sellam.V, Siva Surya Narayanan.V.Y, Karthikeyan Kumar, Anurag, Sudipta Kumar Das
}

\begin{abstract}
The Twitter interpersonal organizations give an approach to clients to keep up contact with others. Expanding of the informal organization's quality allows every one of them to accumulate monstrous measures of nonpublic information with respect to their clients. Tragically, this data riches just as its straightforwardness to get to clients' data could draw in malevolent gathering's consideration. That is the reason these systems have been attacked by spammers while, there have been a great deal of work to analyze and fix them. With reference to this issue spammers look for new ways that to concentrate on these systems every day, there have being nonstop activities to distinguish spammers and malignant email. The point of this paper is to take a gander at past works inside the field of spam identification in informal communities. In late time, on-line interpersonal organizations are loaded with differed undesirable dangers. Despite the fact that they gave us an open stage to impart our contemplations to other people, notwithstanding, because of abuse of this incredible asset, general clients are in jeopardized condition. For instance, YouTube has been utilized as a limited time ground by different craftsman to transfer their music recordings, film trailers, and so on and watchers can post their supposition on them. Tragically, regularly malevolent clients use to post phishing site connections, promotions, and deceitful data in the remarks segment, which may transmit infections or malwares.
\end{abstract}

Keywords: Spamming In Social Media, Hop To N Hop, Os Securities

\section{INTRODUCTION}

We propose an amalgamation of network based highlights with other component classifications for distinguishing mechanized spammers, wherein networks are recognized utilizing chart dividing calculations. favorable clients by and large pursue and react to demands from known clients and maintain a strategic distance from association with and correspondence from outsiders. as such, in the system of trust of a client, most clients display a specific dimension of trust in the personality of others, which prompts the development of a network like structure. a considerate client might be an individual from numerous networks relying upon genuine

Revised Manuscript Received on October 18, 2019

Sellam V, Assistant Professor, SRM IST, Chennail Tamil Nadu, India.

Karthikeyan Kumar, Pursuing B.Tech, Computer Science and Engineering, SRM IST, Ramapuram, Chennai, Tamil Nadu, India. Engineering in SRM IST, Ramapuram, Chennai, Tamil Nadu, India.

Siva Surya Narayanan.V.Y, Pursuing B.Tech, Computer Science and Engineering, SRM IST, Ramapuram, Chennai, Tamil Nadu, India.

Anurag, Pursuing B.Tech, Computer Science and Engineering, SRM IST Ramapuram, Chennai, Tamil Nadu, India.
Sudipta Kumar Das is currently pursuing B.Tech computer Science and

systems and interests. conversely, spammers for the most part pursue arbitrary clients, which results in an incredibly low response rate that shapes meager associations among supporters, and antagonistically influences connection and network based highlights. we propose a half and half

methodology for identifying social spam bots in twitter, which uses an amalgamation of metadata-, content-, collaboration, and network based highlights. in the investigation of portraying highlights of existing ap-

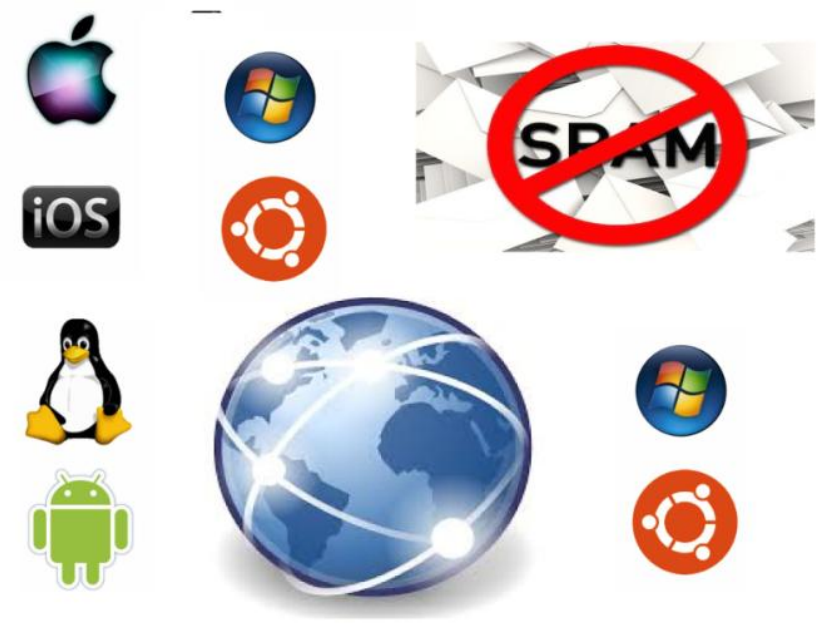

Fig. 1. NanoJ framework. World filled of Operating systems full of data.

proaches, most system based highlights are not characterized utilizing client devotees and basic network structures.we arrange our arrangement of highlights into three general classes, to be specific, metadata, substance, and system.

\section{RELATED WORKS}

Spamming is that the utilization of informing frameworks like messages and option advanced conveyance frameworks and communicate media to send undesirable mass messages aimlessly. The term spamming is furthermore connected to elective media like in web discussions, moment electronic correspondence, and versatile content electronic correspondence, long range interpersonal communication spam, garbage fax transmissions, Television advancing and sharing framework spam. Malicious associations square measure unites made with the intend to hurt, cheat or harm a customer or their device. Right when the association is clicked, practices enacted will move from downloading malware to taking individual data.

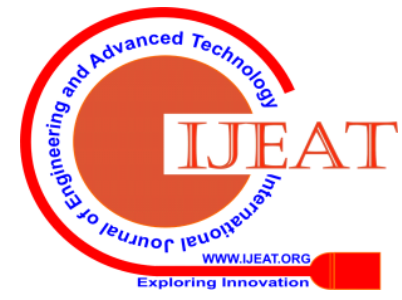




\section{Spamex: A Multi Array Methodology to Detect Computerized Spammers in Twitter Social Media}

\section{A. Spamming In Social Media}

Broadened half breed approach for sleuthing spammers on Twitter, which uses a consequences of consolidating information, content, association, network based and alternatives of existing methodologies, arrange based choices aren't laid out exploitation unmistakable data preparing address based choices. accordingly regardless of the very certainty that the name of client inside a similar data processet al. | bioR $\chi i v$ |March 27, 2019 $1-8$

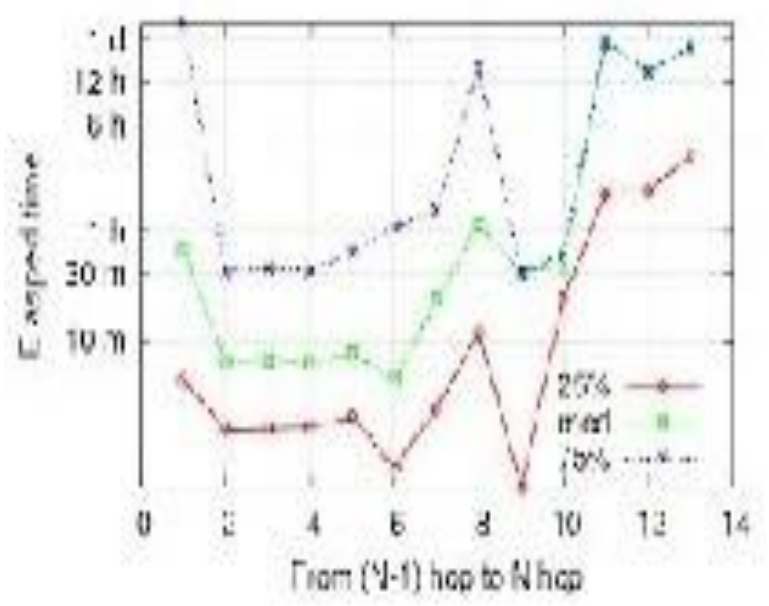

Fig. 2. Hop to n Hop a) Automation has increased over the years.

ing address to associates different records in a solitary framework might be a noxious movement of spammers. Spammers fundamental motivefor spreading imagine messages and undesirable tweet substance share by means of tweets on the network. At that point they have to shape a virtual network in twitter account so exploitation spam bots is that the correct decision for spammers Spam bots square measure making pretty much anyway initiated inside the single machine it's an equivalent data handling address with the different spammer's records. At that point exclusively spammers ought to be recognized with the one of a kind data handling essentially based order. UIP basically based part is convincing technique for isolating monotonous spam bots tweets inside a comparative information getting ready. request set of options into 5 wide classes, to be explicit, metadata, substance, system, and framework, sort out decisions are removed from practical additional data relating to the undeniable information getting ready Address of a customer, however mastermind based features expect to look at specific information taking care of location. Framework based features square measure removed from customer joint effort organize. Metadata alternatives demonstrate the littlest sum sway on the execution of the classifiers, that features the strength of arbitrary number generator calculations, utilized by bots to achieve arbitrariness in their conduct simply like those of human-beings[2]. Different transmitter accounts act in a solitary machine so a similar data handling contains all the transmitter bots. One of a kind IP address fundamentally based data handling order expels intermittent data preparing address address.

\section{B. Other Relevant Tools} organize based alternatives. In the examination of portraying

Robotization of spammers can be distinguished through transient investigation given 0that they utilize arbitrary number generator calculations to fix movement time. Nonetheless, randomization calculations still pursue certain disseminations. Bots are modified to be enacted at a predefined point in time as indicated by the time initiation work. The TSD include is characterized to catch the varieties in tweet times of a client.

where to is the tweet time of the it tweet, $t$ is the mean tweet time, and $\mathrm{N}(\mathrm{u})$ is the all out number of tweets posted by $\mathrm{u}$. The estimation of TSD is commonly very low for robotized spammers. 2.1.4 Tweet Time Interval Standard Deviation (TISD): TISD tracks designs in the time interim of sequential exercises. Bots by and large post tweets at normal time interims. 2.2.3 Mention Ratio (MR): Twitter Users can be labeled in a tweet utilizing the "@" image pursued by their Twitter handles for notice some client. This element is additionally mishandled by spammers who notice clients in tweets, along these lines inciting and tempting them to think about the sender of the message. This nature makes generous clients vulnerable to unfortunate casualties. $\mathrm{z}$ Where $\mathrm{M}(\mathrm{u})$ is the quantity of notices in the tweets and $N(u)$ is the quantity of tweets posted by u.In general, the estimation of the MR include is low for benevolent clients and high for spammers. 2.2.4 Unique Mention Ratio (UMR): In Twitter Users for the most part have associations with various individuals, including companions, relatives, and partners. () Evidently, the estimation of UMR is low for certifiable clients given that they collaborate with a chose set of individuals, while it is high for spammers. In the event that spammer focuses on a particular arrangement of clients by consistently referencing them in tweets, at that point the spammer will be seen and revealed by the client, and the vindictive aim of the spammer will be uncovered. To watch the conduct of the two classes of clients, the combined. (1).

\section{Content And Hashtag Similarity (Chs)}

Spammers abuse the slanting subjects recorded by Twitter by infusing them into their malignant tweet substance. Despite the fact that spammers infuse drifting hashtags into their Direct Messages, these hashtags and tweets content have no semantic connection. Because of hashtag infusion, at whatever point favorable client look tweets that relate to an inclining hashtag, vindictive tweets infused with that hashtag will likewise be shown in the sought outcome, accordingly raising the likelihood of the client turning into a casualty of spamming. The CHS highlight is characterized to catch such run of the mill social building strategies utilized by spammers. ()$=()=1()$ Where $\mathrm{M} \mathrm{Hi}(\mathrm{u})$ is the quantity of words that coordinate with the hashtags utilized in the ith tweet of client $\mathrm{u}, \mathrm{HT} \mathrm{i}(\mathrm{u})$ is the quantity of hashtags utilized in the ith tweet, and $\mathrm{N}(\mathrm{u})$ is the complete number of tweets by $\mathrm{u}$. The estimation of CHS is commonly high for favorable clients given that their hashtags and tweet subjects are commonly the equivalent, spammers and benevolent clients don't significantly unique as far as CHS and 80than 0.2. 2.2.6 Hashtag Ratio (HTR): Hashtags in Twitter are utilized to 
aggregate tweets identified with any theme of talk. Not at all like different OSNs, for example, Facebook, where bunches are made with the name speaking to the subject of intrigue. A gathering for talking about a subject of intrigue is made in Twitter through hashtags. Twitter shows a rundown of the main 10 drifting hashtags at any minute. This component is abused by spammers by commandeering these inclining themes. Spammers infuse prominent hashtags into their tweets, with the end goal that at whatever point these hashtags are looked, tweets by the spammers that contain the sought hashtags are additionally appeared in the item. The HTR for client $u$ is characterized. () HT(u) is the quantity of hashtags utilized in the tweets and $\mathrm{N}(\mathrm{u})$ is the all out number of tweets posted by $\mathrm{u}$. When all is said in done, the estimation of HTR for spammers is high, while it is low for amiable clients. 2.2.7 Automated Tweet URL Ratio (AUR): To catch content quality in the computerized tweets of clients, this element is exceedingly significant on the grounds that it examines the utilization of URLs in robotized tweets. The AUR of client $u$ is characterized as the proportion of.

\section{Os Securities}

This study of existing ways for sleuthing spam profiles in OSNs has been done once a logical survey with high-principled methodology amid which significant investigation databases for pc Science are looked like IEEE Xplore, ACM Digital Library, SpringerLink, Google Scholar, ScienceDirect for concerned subject. we watch out for focussed on papers once year 2009 exclusively as the possibility of interpersonal organizations appeared exclusively in 1997 [1] and have turned out to be basic exclusively later. At that point Facebook was propelled inside the year 2004 [1] that turned out to be very prevalent. So it required your investment for people to initiate familiar with these systems for correspondence and in this way the assaults on these systems.

This inquiry from higher than referenced five noteworthy databases returned more than sixty papers. Papers looked into for this review paper were chosen once perusing titles and modified works of the considerable number of papers. just those papers were picked that were discovered suitable for the present examination. Papers with titles and modified works identifying with spam messages discovery and option rude points square measure avoided for the present paper in this manner at last a total of twenty one papers are chosen for survey. essentially the papers are arranged on the possibility of choices wont to watch spammers. Through this paper we tend to attempt to order a posting of long range interpersonal communication papers on discovery of spam profiles in Twitter that we have examine. The rundown could without a doubt be fragmented, however gives [11:49, 3/21/2019] Surya: structure to this examination incorporating interpersonal organization spammer location. When surfing this study paper, new analysts will just gauge what work has been done, in which year and the manner in which this work will be reached out to make spam identification extra right. At whatever point material, we have. identification of spammers and precision In explicit, the papers cowl yet spammers move with informal organization clients, their suggestions and existing methods to locate these spammers. 3. SECURITY issues IN OSNs Online Social Networking locales (OSNs) ar in danger of security and protection issues as an aftereffects of the amount of client data being prepared by these destinations step by step. Clients of long range informal communication locales ar presented to different assaults: 1) Viruses - spammers utilize the interpersonal organizations as a stage [19] to unfurl pernicious information at interims the arrangement of clients. 2) Phishing assault client's touchy information is acquired by mimicking a dependable other gathering [30]. 3) Spammers - send awful spam messages to the clients through informal communities [11]. 4) Sybil assault - awful individual gets various phony personalities and professes to be genuine at interims the framework so as to make harm the name of legitimate clients at interims the system [20]. 5) Social bots-a gathering of false profiles that ar made to collect clients' close to home information [32]. 6) Clone and misrepresentation assaults where aggressors turn out a profile of officially existing client at interims indistinguishable system or crosswise over various systems subsequently on trick the cloned client's companions [23]. In the event that exploited people acknowledge the companion demands sent by these cloned characters, at that point assailants square measure ready to get to their data. These assaults devour additional assets from clients and frameworks. 4. types of Spammers ar the pernicious clients managerial body pollute the data exhibited by genuine clients and thusly cause a hazard to the security and protection of informal organizations.

Spammers have a place with at least one in everything about after classes [22]: 1 . Phishers: ar the clients regulatory body act sort of a standard client to obtain individual information of other genuine clients. 2. counterfeit Users: ar the clients managerial body imitate the profiles of genuine clients to send spam substance to the companions' of that client or different clients at interims the system. 3. Advertisers: ar those regulatory body send malevolent connections of notices or different special connects to other people so on procure their own information. Thought processes of Spammers: a) clear creation b) unfurl infections c) Phishing assaults.

Windows: The Windows stage has roused a similarly specially appointed application framework, with outsider application delicate product being unremarkably nonheritable from various to shield themselves against malware, which might be a well-reported drawback for Windows. Macintosh: In qualification, the mack stage is for the most part seen to be progressively verification against malware, as there are nearly less reported instances of malware assaults. Macintoshes even have hostile to infection operation tions, anyway they're less wide received [17]. practically like Windows, the customary application plot has conjointly been generally decen-tralized. prompted by the accomplishment of the portable App Store, Apple propelled the mackintosh App Store as an incorporated commercial center for work area applications. It is by all accounts genuinely prosperous [1]. Android: There square measure many "commercial centers" for robot clients to download applications, with the robot Market being the first well known. The robot Market isn't 


\section{Spamex: A Multi Array Methodology to Detect Computerized Spammers in Twitter Social Media}

curated, however ongoing re-ports counsel that it's checked for malware by Google [2]. (Google additionally expels bundle that is found to abuse their TOS.) There are numerous incontestable malware assaults on the robot stage.

Hostile to infection applications square measure realistic for robot, however their ef-fectiveness has been in open addressed [33, 29]. iOS: The App Store could be a brought together, curated commercial center for down-stacking iPhone applications. though the exact subtleties of the curation procedure is unknown, 1 there is confirmation to guide that Apple will check for security infringement. in spite of the fact that there are not many sam-ples of iPhone malware, there's numerous grayware and escape ing applications [23]. Clients square measure provoked once applications wish to get to area or elective information through popup warnings.

Broadened crossover approach for sleuthing spammers on Twitter, which uses a consequences of joining information, content, association, network based and arrange based choices. In the examination of describing choices of existing methodologies, arrange based alternatives aren't laid out exploitation particular data preparing address based choices. in this manner regardless of the very truth that the name of client inside a similar data handling address to associates different records in a solitary framework might be a malignant movement of spammers. Spammers primary motivefor spreading imagine messages and undesirable tweet substance share by means of tweets on the network. At that point they have to shape a virtual network in twitter account so exploitation spam bots is that the correct decision for spammers Spam bots square measure making pretty much anyway actuated inside the single machine it's an equivalent data preparing address with the numerous spammer's records. At that point exclusively spammers ought to be identified with the exceptional data preparing essentially based order. breakdown.jpg

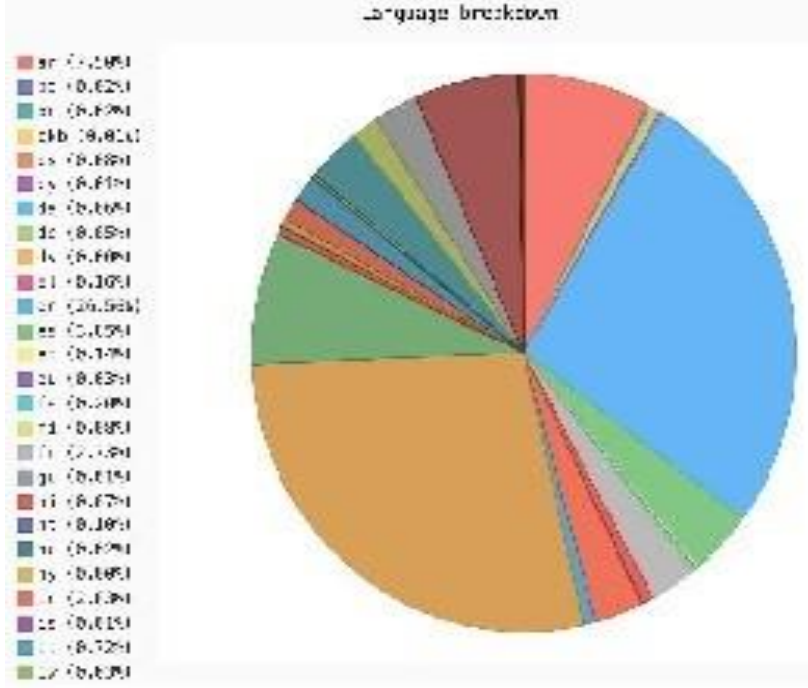

Fig. 3. Language breakdown

UIP basically based element is compelling technique for separating intermittent spam bots tweets inside a similar data handling. arrange set of alternatives into 5 expansive classes, to be specific, metadata, substance, network, and system, organize choices are extricated from possible further information identifying with the unmistakable data preparing Address of a client, though organize based highlights mean to take a gander at particular data handling address. System based highlights square measure removed from client connection arrange.

Proposed Systems. In computer network, there ar vendors that allows you to gain followers at all-time low cost 7 , like 18000 followers for 15 dollars solely. Spammers usually manage followers through mutual consent by following one another to evade network connected options. number of followers as of benign users. This finding indicates that typical options, like variety of followers, followers to followings magnitude relation, retweet magnitude relation supported user knowledge are insufficient for development of effective sender detection systems. in contrast, community-, follower-, and content- based options, outlined exploitation user and his/her followers knowledge enable the event of effective sender detection systems. though metadata-based options ar usually least contributing in sender detection thanks to the very fact that spammers exploit organization algorithms to imitate randomness for benign users on condition that they typically retweet the tweets of others. The experimental results shown within the preceding sections replicate that freshly outlined interaction-based options based on followers area unit extremely effective in detective work social spambot. Interaction-based options also are balanced in terms of each FPR and F-Score values. additionally, community- based options additionally show important performance and aid in the improvement of the classifiers accuracy. In summary,it is concluded that followers and communities from the interaction network of user area unit robust indicators of the name of users. Features supported these classes is used for economical segregation of social spambots and benign users, in distinction to conventional user-centric options. 1)ration of spammers and benign users on the analysis results. To this finish, we tend to perennial the experiment for various ration of spammers and benign users viz 1:1, 1:2, 1:5, and 1:10 and presented the analysis leads to terms of the 3 analysis metrics, namely, DR, FPR, and F-Score for the classifiers - random forest, call tree, and Bayesian network in Table V. The dataset with spammers and benign users ration as 1:1 has one thousand spammers and one thousand benign users, whereas dataset with $1: 2$ ration has five hundred spammers and one thousand benign users. In the analysis, dataset magnitude relation shows correlation with the potency metrics except the Bayesian network wherever the correlation is not important and magnitude relation of spammers and benign users within the dataset doesn't show important impact on the result. In the case of random forest and call tree, as we tend to decrease the ratio of spammers within the dataset, accuracy of the classifiers also decreases, that is very important just in case of random forest. during this case, the worth of DR considerably decreases ondecreasing the spammers within the dataset as shown within the Table V. This decrease within the performance of the classifiers could also be due to category imbalance downside. 2) applied math Significance Analysis of the behavioural Dif- ference of Spammers and Benign Users: This section presents a applied math significance analysis to answer the question: "is the distinction between the operating behavior of spammers and benign users in terms of freshly outlined options a

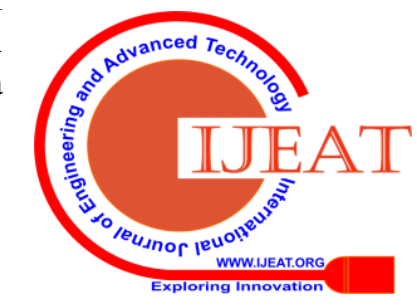


random chance?" to check this hypothesis, we have a tendency to perform two-tailed Z- test victimisation the dataset mentioned in Section III-A. In Z-test,

under invalid theory, investigate insights is intended to pursue the ordinary circulation, and is assessed exploitation 2 speculations - invalid theory ( $\mathrm{H} 0: \mu=\mu 0)$ and different theory $(\mathrm{H} 1: \mu 6=\mu 0)$ [34]. In invalid theory, supposition that will be that there's no essential distinction inside the populace proposes that of newly plot include values among spammers and kindhearted clients, though in elective speculation, we tend to accept importance of the component estimations of the 2 classifications differ significantly. From there on, investigate measurements for every one of the about six crisply sketched out and a couple of reclassified choices is determined and contrasted and the arranged significant estimations of two-followed Z-insights at five-hitter criticalness level, that is \pm 1.96 . In the investigation, invalid speculation for all the eight choices is dismissed as appeared Table VI. In this way, it's finished that mean estimations of all the eight choices for spammers and favorable clients differ significantly. As clear from the table, mean The strategy arranged amid this investigation assesses a particular client at the time they mastermind to be a piece of a sub-network, which is

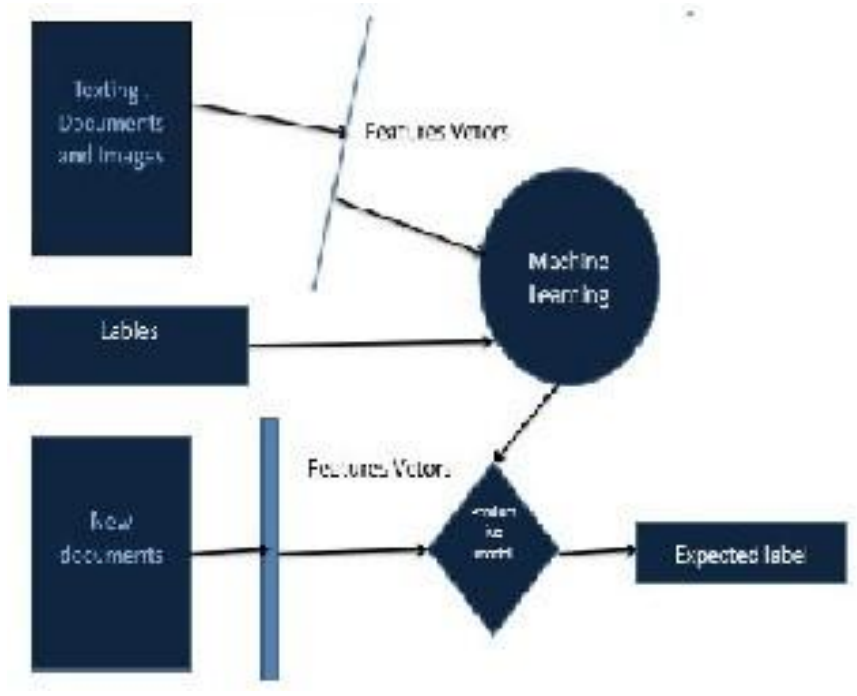

Fig. 4. AI AUTOMATION WITH LABELS AND ML

gave the impression to be this date. For testing capacities, all dates were balanced in order to recreate the time once accounts attempted to append the sub-network of intrigue. Basically, for each one among the 838 records inside the example, Algorithm one was wont to create a system at any given moment set by the daterestriction facultative parameter. This technique is an especially fundamental a piece of structure a right training set. When the instructing set is made, the time used for each new client that makes an endeavor to attach a network is foreseen to be the present date. The dates that would be set for the training dataset fluctuated for the genuine records and illicit clients. Since thoughtfully, misdirection prevention makes an endeavor to pass judgment and render a decision for a client at the season of attempted passage amid a network,

After execution the aforementioned methodology, each so-cial organize metric referenced inside the past area was determined for each client. for instance, client A might be a restricted client and made their first post on giftcardexchange at Tentry time. algorithmic program one is named abuse the resulting param-eters: CommonContributionNetwork(A, Tentry). The outcome creates a fix of the sub-network's system with the denied client encased in that shot.

Proportion of spammers and generous clients on the examination results. To this completion, we watch out for enduring the test for different apportion of spammers and considerate clients viz $1: 1,1: 2,1: 5$, and $1: 10$ and displayed the investigation prompts terms of the 3 examination measurements, to be specific, DR, FPR, and F-Score for the classifiers - arbitrary woods, call tree, and Bayesian system in Table V. The dataset with spammers and amiable clients proportion as 1:1 has one thousand spammers and one thousand benevolent clients, while dataset with 1:2 apportion has five hundred spammers and one thousand amiable clients. In the examination, dataset extent connection demonstrates connection with the power measurements aside from the Bayesian system wherever the connection isn't significant and greatness connection of spammers and kindhearted clients inside the dataset doesn't indicate significant effect on the outcome. On account of irregular woods and call tree, as we will in general diminishing the proportion of spammers inside the dataset, exactness of the classifiers likewise diminishes, that is significant just in the event of arbitrary woodland. amid this case, the value of DR impressively diminishes on diminishing the spammers inside the dataset as appeared inside the Table V. This reduction inside the execution of the classifiers could likewise be because of classification awkwardness drawback. 2) connected math Significance Analysis of the social Dif-ference of Spammers and Benign Users: This area displays a connected math hugeness investigation to address the inquiry: "is the qualification between the working conduct of spammers and amiable clients regarding crisply laid out alternatives an arbitrary possibility?" to check this speculation, we tend to perform twotailed Z-test exploitation the dataset referenced in Section III-A. In Z-test,under invalid speculation, investigate measurements is intended to pursue the typical dissemination, and is assessed exploitation 2 hypotheses- invalid theory ( $\mathrm{H} 0: \mu=\mu 0)$ and different speculation $(H 1: \mu 6=\mu 0)$ [34].In invalid theory, supposition that will be that there's no indispensable distinction inside the populace proposes that of crisply plot include values among spammers and kind users, whereas in elective speculation, we tend to accept importance of the element estimations of the 2 classifications differ significantly. From there on, investigate measurements for every one of the about six naturally laid out and a couple of re-imagined choices is determined and contrasted and the organized significant estimations of two-followed Z-insights at five-hitter hugeness level, that is \pm 1.96 . In the investigation, invalid theory for all the eight alternatives is dismissed as appeared Table VI. Hence, it's finished that mean estimations of all the eight choices for spammers and kind clients differ impressively. As apparent from the table, mean The strategy arranged amid this investigation assesses a particular client at the time they organize to be a piece of

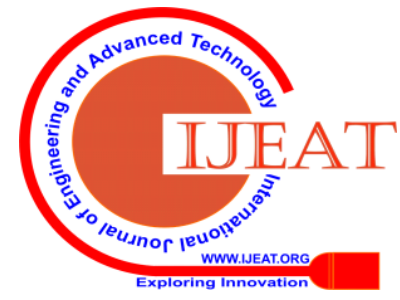




\section{Spamex: A Multi Array Methodology to Detect Computerized Spammers in Twitter Social Media}

a sub-network, which is gave the impression to be this date. For testing functions,all dates were balanced in order to reproduce the time once accounts attempted to attach the sub-network of intrigue. Basically, for each one among the 838 records inside the example, Algorithm one was wont to produce a system at any given moment set by the daterestriction facultative parameter. This technique is an especially important a piece of structure a right training set. When the training set is made, the time used for each new client that makes an endeavor to fasten a network is foreseen to be the present date. The dates that would be set for the training dataset changed for the real records and illicit clients. Since adroitly, double dealing prevention makes an endeavor to pass judgment and render a decision for a client at the season of attempted passage amid a community,After execution the aforementioned methodology, every informal organization metric referenced inside the past area was determined for each client. for instance, client A might be a restricted client and made their first post on giftcardexchange at Tentry time. algorithmic program one is named misuse the consequent parameters: CommonContributionNetwork(A, Tentry). The outcome produces a dose of the sub-network's system with the disallowed client encased in that shot. For client A (presently a hub inside the system) the ensuing are determined..

\section{PROPOSED METHODS}

Boxplots depiction the variations between illegal accounts (de-ceivers) and bonafide users at Tentry (time of entry within the sub-community) and Tbanned (time of being illegal by the sub-community). Social network metrics embrace degree (CD), closeness (CC ), betweeness (CB), eigenvector centrality $(\mathrm{CE})$, eccentricity (e) and constraint (C). CD(A), CC (A), CB(A), CE(A), e(A) and C(A). Once the same procedure is applied for all users, the ultimate sample contained legitimate and illegal users beside their social network metrics within the explicit network pic in time (Tentry or Tbanned). Fig. a pair of depicts the variations between these metrics and respective teams of users. These were calculated for illegalaccounts (henceforth deceivers) at Tentry and Tbanned additionally as legitimate accounts at Tentry. it's evident that there's a visual distinction between the cluster of illegal users and therefore the legitimate users.

Deceivers doubtless have a harder time constructing a standard contribution network that relates well to existing members of the sub-community. this could be due to an absence of focus since illegal accounts might haven't originally supposed to be a part of the actual sub-community.Another reason is that deceivers might not have allowed enough time for connections to be generated. any discussion on these results is obtainable at a later section of this paper.Deceivers seem to own substantial variations in social network metrics compared to legitimate users shown on fig. 2. Metrics indicate that deceivers area unit overall any apart in terms of however they slot in the community, compared to legitimate users. The impact looks to be higher once measurements area unit made at the time of entry (during the primary post within the subcommunity) compared to the time a user was illegal

\section{MANUAL ANALYSIS}

Tbanned (time of being illegal by the subcommunity). (i, j) $=\log 2 \mathrm{R}(\mathrm{ui}, \mathrm{uj}) \times \mathrm{I}(\mathrm{uj} \mid \mathrm{ui})+1$ Boxplots depiction the variations between illegal accounts (de- ceivers) and bonafide users at Tentry (time of entry within the sub-community) and Tbanned (time of being illegal by the sub-community). Social network metrics embrace degree (CD), closeness (CC ), betweeness (CB), eigenvector centrality $(\mathrm{CE})$, eccentricity (e) and constraint (C).CD(A), $\mathrm{CC}(\mathrm{A}), \mathrm{CB}(\mathrm{A}), \mathrm{CE}(\mathrm{A}), \mathrm{e}(\mathrm{A})$ and $\mathrm{C}(\mathrm{A})$. Once the same procedure is applied for all users, the ultimate sample contained legitimate and illegal users beside their social network metrics within the explicit network pic in time (Tentry or Tbanned). Fig. a pair of depicts the variations between these metrics and respective teams of users. These were calculated for illegal accounts (henceforth deceivers) at Tentry and Tbanned additionally as legitimate accounts at Tentry. it's evident that there's a visual distinction between the cluster of illegal users and therefore the legitimate users. Deceivers doubtless have a harder time constructing a standard contribution network that relates well to existing members of the sub-community. this could be due to an absence of focus since illegal accounts might haven't originally supposed to be a part of the actual sub-community. Another reason is that deceivers might not have allowed enough time for connections to be generated. any discussion on these results is obtainable at a later section of this paper. Deceivers seem to own substantial variations in social network metrics compared to legitimate users shown on fig.2. Metrics indicate that deceivers area unit overall any apart in terms of however they slot in the community, compared to legitimate users. The impact looks to be higher once measurements area unitmade at the time of entry (during the primary post within the subcommunity) compared to the time a user was illegal.

\section{A. Discussion And Future Perspectives}

In this paper, we've planned associate degree approach to spot a hierarchic list of radically authoritative users in net forums. We have developed a radicalness live and a spread of collocationbased association measures, and designed an algorithmic rule supported PageRank to rank the radically influential users. Among the planned association measures, the contingency constant live is found because the most promising live, once embedded within the custom-made PageRank algorithmic rule beside the radicalness live. The experimental results on a typical knowledge set square measure promising that outperforms the prevailing UserRank algorith mic rule. It is also found that the collocation-based association measures deal with such ranking drawback additional effectively than matter and temporal similarity primarily based measures. This work opens many promising directions for future research. 


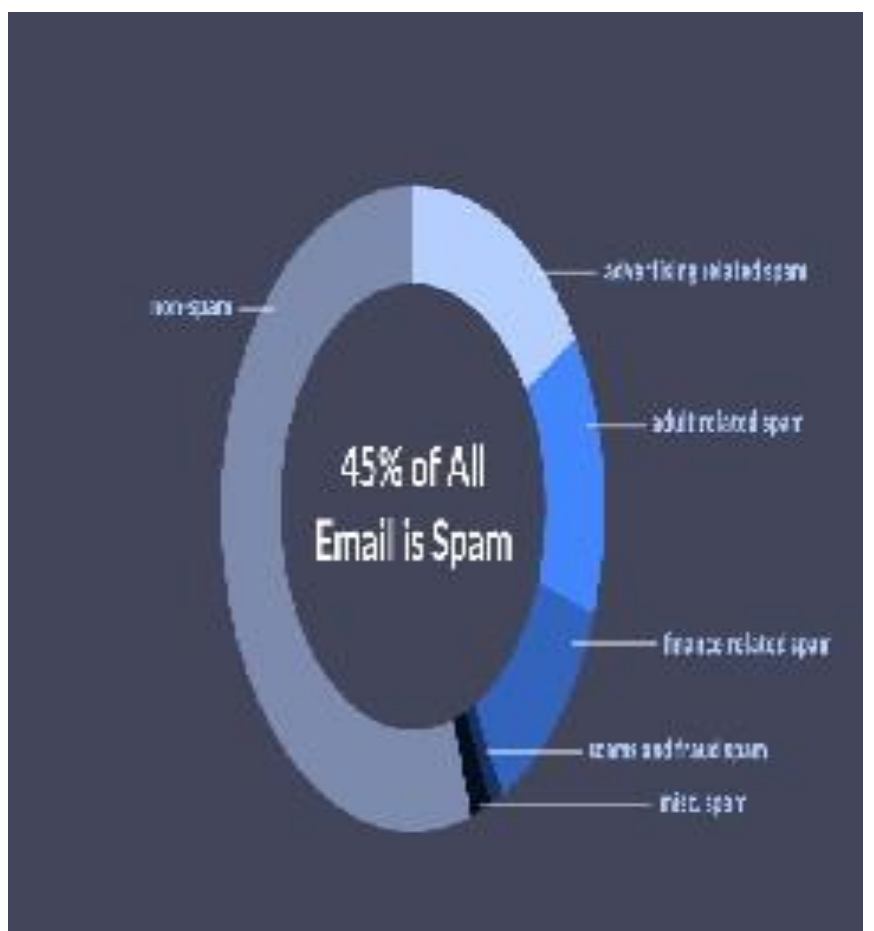

FIG. 5. SPAM CONTENTS IN A SPAM MESSAGE

gave the impression to be this date. For testing capacities, all dates were balanced in order to recreate the time once accounts attempted to join the sub-network of intrigue. Basically, for each one among the 838 records inside the example, Algorithm one was wont to create a system at any given moment set by the daterestriction facultative parameter. This method is an especially vital a piece of structure a right instructing set. When the training set is made, the time used for each new client that makes an endeavor to append a network is foreseen to be the present date. The dates that would be set for the instructing dataset changed for the real records and unlawful clients. Since theoretically, trickiness block makes an endeavor to pass judgment and render a decision for a client at the season of attempted passage amid a network,

After execution the aforementioned system, each so-cial arrange metric referenced inside the past segment was determined for each client. for instance, client A might be a precluded client and made their first post on giftcardexchange at Tentry time. algorithmic program one is named abuse the ensuing param-eters: CommonContributionNetwork(A, Tentry). The outcome creates a fix of the sub-network's system with the denied client encased in that shot.

\section{CONCLUSION}

Considering social relations also to the strung collaborations, investigating etymology factors like exchange setting and subject float for profundity distinguishing proof, and applying feeling examination to separate between the clients taking positive and negative sides of fundamentalism, are not many crucial examination issues. Breaking down the affect of The development of online networking applications is by all accounts happening at Associate in Nursing unpresented rate, that has brought about Associate in Nursing expanded enthusiasm for trademark novel manners by which to fight character misleading and particularly personality phony. Be that as it may, in spite of the endeavors and deterrent methods known by designers, developers, and scientists, duplicity impediment has not been given enough consideration. This examination offers an interesting imagine to exhibit a procedure way to deal with personality misleading anticipation by using interpersonal organization data and explicitly a typical commitment arrange.

Software and Hardware Availability. Spamming requires not more than a computer with basic facilities to build a bot and spam and load a site full of garbage.

- https://github.com/bazhenov/twitter-spam-detector

- https://github.com/jasleenkaur94/Spam-detection-on-Twitte $r$

\section{REFERENCES}

1. Michael J Mlodzianoski, John M Schreiner, Steven P Callahan, Katarina Smolková, Andrea Dlasková, Jitka Šantorová, Petr Ježek, and Joerg Bewersdorf. Sample drift correction in $3 \mathrm{~d}$ fluorescence photoactivation localization microscopy. Optics express, 19(16):15009-15019, 2011.

2. T. Anwar and M. Abulaish, "Ranking radically influential web forum users," IEEE Transactions on Information Forensics and Security,vol. 10 no. 6, pp. 1289-1298, 2015.

3. Y. Boshmaf, I. Muslukhov, K. Beznosov, and M. Ripeanu, "Design and analysis of social botnet," Computer Networks, vol. 57, no. 2, pp. 556-578, 2013.

4. D. Fletcher, "A brief history of spam,” TIME, Tech. Rep., 2009.

5. Y. Boshmaf, M. Ripeanu, K. Beznosov, and E. Santos-Neto, "Thwarting fake osn accounts by predicting their victims," in Proc. AISec., Denver,2015, pp. 81-89.

6. N. R. Amit A Amleshwaram, S. Yadav, G. Gu, and C. Yang, "Cats:Characterizing automation of twitter spammers," in Proc. COMSNETS, Banglore, 2013, pp. 1-10.

7. K. Lee, J. Caverlee, and S. Webb, "Uncovering social spammers: Social honeypots + machine learning," in Proc. SIGIR, Geneva, 2010, pp 435-442.

8. G. Stringhini, C. Kruegel, and G. Vigna, "Detecting spammers on social networks," in Proc. ACSAC, Austin, Texas, 2010, pp. 1-9.

9. K. Vijayakumar, S. Suchitra and P. Swathi Shri, "A secured cloud storage auditing with empirical

10. outsourcing of key updates", International Journal Reasoning-based Intelligent Systems, Vol. 11, No. 2, 2019.

11. G. Indrajith and K.Vijayakumar, "Automatic Mathematical and Chronological Prediction in Smartphone Keyboard" International Journal of Engineering and Computer Science ISSN: 2319-7242Volume 5 Issue 5 May 2016, Page No. 16714-16718.

12. K. Vijayakumar and C. Arun, "A Survey on Assessment of Risks in Cloud Migration", International Journal of Applied Engineering Research, ISSN 0973-4562 Vol. 10 No.66 May 2015.

\section{AUTHORS PROFILE}

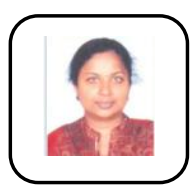

Sellam V is currently working as Assistant Professor in SRM IST, Chennail Tamil Nadu. She has completed her B.E and M.E in Computer Science and Engineering from Anna University, Chennai, Tamil Nadu

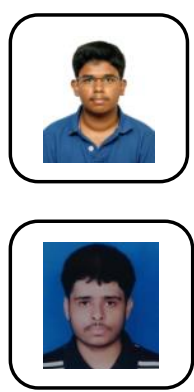

Karthikeyan Kumar is currently pursuing B.Tech computer Science and Engineering in SRM IST, Ramapuram, Chennai, Tamil Nadu, India.

Sudipta Kumar Das is currently pursuing B.Tech computer Science and Engineering in SRM IST, Ramapuram, Chennai, Tamil Nadu, India.

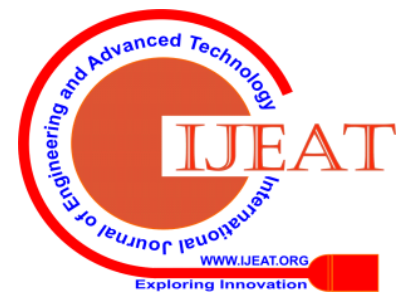


Spamex: A Multi Array Methodology to Detect Computerized Spammers in Twitter Social Media

Siva Surya Narayanan.V.Y is currently pursuing B.Tech computer Science and Engineering in SRM IST, Ramapuram, Chennai, Tamil Nadu, India.

Anurag is currently pursuing B.Tech computer Science and Engineering in SRM IST, Ramapuram, Chennai, Tamil Nadu, India. 